\title{
A Professional Development Model for Medical Laboratory Scientists Working in the Immunohematology Laboratory
}

\author{
MELINDA N. GARZA, LILA A. PULIDO, MEGAN AMERSON, FAHEEM A. ALI, BRANDY A. \\ GREENHILL, GARY GRIFFIN, ENRIQUE ALVAREZ, MARSHA WHATLEY, AND PETER C. HU
}

\section{ABSTRACT}

Transfusion medicine, a section of the Department of Laboratory Medicine at The University of Texas MD Anderson Cancer Center is committed to the education and advancement of its health care professionals. It is our belief that giving medical laboratory professionals a path for advancement leads to excellence and increases overall professionalism in the Immunohematology Laboratory. As a result of this strong commitment to excellence and professionalism, the Immunohematology laboratory has instituted a Professional Development Model (PDM) that aims to create Medical Laboratory Scientists (MLS) that are not only more knowledgeable, but are continually striving for excellence. In addition, these MLS are poised for advancement in their careers. The professional development model consists of four levels: Discovery, Application, Maturation, and Expert. The model was formulated to serve as a detailed path to the mastery of all process and methods in the Immunohematology Laboratory. Each level in the professional development model consists of tasks that optimize the laboratory workflow and allow for concurrent training. Completion of a level in the PDM is rewarded with financial incentive and further advancement in the field. The PDM for Medical Laboratory Scientists in the Immunohematology Laboratory fosters personal development, rewards growth and competency, and sets high standards for all services and skills provided. This model is a vital component of the Immunohematology Laboratory and aims to ensure the highest quality of care and standards in their testing. It is because of the success of this model and the robustness of its content that we hope other medical laboratories aim to reach the same level of excellence and professionalism, and adapt this model into their own environment.

ABBREVIATIONS: PDM, professional development model; MLS, medical laboratory scientist
INDEX TERMS: Professional, Model, Development

Clin Lab Sci 2012;25(1):2

Melinda N. Garza, School of Health Professions, The University of Texas, MD Anderson Cancer Center, Houston, TX 77030

Lila A. Pulido, Division of Pathology and Laboratory Medicine, The University of Texas, MD Anderson Cancer Center, Houston, TX 77030

Megan Amerson, School of Health Professions, The University of Texas, MD Anderson Cancer Center, Houston, TX 77030

Faheem A. Ali, School of Health Professions, The University of Texas, MD Anderson Cancer Center, Houston, TX 77030

Brandy A. Greenhill, School of Health Professions, The University of Texas, MD Anderson Cancer Center, Houston, TX 77030

Gary Griffin, Division of Pathology and Laboratory Medicine, The University of Texas, MD Anderson Cancer Center, Houston, TX 77030

Enrique Alvarez, Division of Pathology and Laboratory Medicine, The University of Texas, MD Anderson Cancer Center, Houston, TX 77030

Marsha Whatley, Division of Pathology and Laboratory Medicine, The University of Texas, MD Anderson Cancer Center, Houston, TX 77030

Peter C. Hu, School of Health Professions, The University of Texas, MD Anderson Cancer Center, Houston, TX 77030 


\section{CLINICAL PRACTICE}

Address for Correspondence: Peter Hu, PhD, 1515 Holcombe Blvd, Unit 2, Houston, TX 77030, 713-5633095,pchu@mdanderson.org

\section{INTRODUCTION}

In accordance with the mission of MD Anderson and the core values of education, prevention, and care, the current PDM used in the Immunohematology Laboratory much like our original cytogenetic technology $\mathrm{PDM}^{1}$, is composed of four different levels of progression: Discovery, Application, Maturation, and Expert. (Table1-4) The PDM in the Immunohematology Laboratory aims to help the Medical Laboratory Scientist (MLS) achieve full competency at each level. To be fully competent at each level, the MLS must demonstrate the capacity to complete the tasks for that level as described in the PDM. Competency is defined as the ability of the MLS to be able to assimilate the knowledge acquired from the PDM along with improvements in attitude, skills, and behavior. ${ }^{2}$ All these measures are to be used at the laboratory manager's discretion to fit the technical demands required.

\section{Advancement Through the Competency Levels}

Every new MLS in the Immunohematology Laboratory begins in the Discovery level of the PDM. To successfully progress through each level, the MLS must learn the skills required for each level, meet continuing educational requirements, complete assigned projects and tasks, and obtain particular certifications. There is a minimum of four hours of educational benchmarks required per year while under the PDM. The educational benchmarks include continuing education pertaining to the Immunohematology field in the form of online activities, attending lectures, etc. Each competency level in the PDM is designed with a minimum and maximum time frame allowed for completion. To master each competency level, the MLS must go beyond simply learning the task and show successful application of the skills learned. The MLS must also be able to adapt and use critically thinking skills to troubleshoot problems in the laboratory. The time frame allotted for completion of each competency level may be adjusted at the discretion of the laboratory manager on the basis of an employee's prior experience and mastery of all competencies associated with a competency level. Table 1 depicts the allotted completion times for each competency level. Currently, the average time for completion in the Discovery phase of the PDM is 14 months, while for the Application and Maturation phases the average time frame of completion is 23 months.

Each MLS in the Immunohematology Laboratory must successfully complete the Discovery through Maturation competency levels, including associated required certifications within their allotted time frames. MLS who do not comply with these requirements may be disciplined, up to and including termination. The consequences are determined on the basis of the reasons for the MLS not meeting the completion deadline and any special circumstances known to the laboratory manager. Once the MLS completes the Maturation competency level, his/her job title will be Senior Medical Laboratory Scientist. The Expert competency level in the PDM is optional but highly recommended. This is the highest level in the PDM and is intended for those looking to move into supervisory positions. A MLS that has been employed with MD Anderson for 20 years or more has the option of being exempt from any further stipulations in the PDM. However, a MLS who has chosen to be grandfathered in from the PDM is no longer eligible to receive any salary-related adjustments specified in the PDM.

\section{Implementing the Professional Development Model}

The laboratory manager or designee is to meet with each MLS in the Immunohematology Laboratory to review his/her PDM timetable and training schedule on a bi-annual basis. The PDM training plans and performance criteria are described in Tables $1-4$. At the Discovery level (Table 1), the MLS is introduced to the basic practices of blood banking. This includes quality control, type and screen procedures, equipment maintenance, cross-matching, antibody identification, specimen processing and mandatory attainment of MLS certification. In addition, the MLS is required to complete a minimum four hours of educational benchmarks per year, and required to select two areas of interest for specialized training. At the Application level (Table 2), the MLS studies additional blood banking skills and instrumentation; the MLS again is also expected to meet a minimum of four hours of educational benchmarks per year, perform a special project, select two new optional areas of focus in the laboratory for training, and practice communication development. At the Maturation level (Table 3), the MLS must again complete the minimum hours of educational benchmarks per year, investigate and 


\section{CLINICAL PRACTICE}

troubleshoot problems, complete a second project, demonstrate mastery of skills and demonstrate independence through training others. In addition, it is in the Maturation phase that the MLS may choose to advance by attaining specialist certifications or a Master's degree as part of the educational benchmarks required. The advanced certifications are highly encouraged but optional based on each MLS professional motivations. The advanced certifications are not required to complete the level in the PDM.

Table 1. Competency Requirements in the Discovery Phase of the Professional Development Model

$\begin{array}{cc}\text { EDUCATIONAL BENCHMARK } & \\ \text { Mandatory } & \text { Optional } \\ 4 \mathrm{hr} & >4 \mathrm{hr}+\end{array}$

Basic Certification

Blood Bank (BB), Medical

Technologist (MT)

\section{PERFORMANCE AREA}

\section{Mandatory}

Crossmatch Workstation

Antibody Identification

Elution \& Direct Antiglobulin Testing (DAT)

Antigen Typing

Transfusion Reactions

Cold Agglutinin

Daily Reagent Quality Control (QC)

Emergency Release of Blood

REES Temperature Monitoring System

Deviations Reporting

Change Control Process

Blood Component Returns

Basic Troubleshooting \& Reporting (Equip.)

Quarantine and Disposition

Proficiency Testing Process

Basic Component Inventory-Labeling and Irradiation

Specimen Receiving \& Processing

Surgery Schedule Process

Reagent Receiving Process

\section{OPTIONAL PERFORMANCE AREA}

2 Areas (Based on Business Needs)

Nucleic Acid Testing (NAT) (counts as 2)

ORTHO Diagnostics (2,3 shift only, counts as 2)

BIOARRAY (counts as 2)

Flow Cytometry

Galileo (Automated Blood Analyzer)

QC/Preventive Maintenance (PM) (Daily, Weekly \& Monthly)

Infectious Disease Specimen Processing

Supply Chain Process

Parathyroid testing

Send Out testing procedures

Blood Distribution
Finally, at the Expert level (Table 4), which is highly encouraged, but not required, the MLS must complete courses in management, staffing, training, and must meet the minimum hours of mandatory education benchmarks per year in addition to performing various leadership activities.

Table 2. Competency Requirements in the Application Phase of the Professional Development Model

\section{EDUCATIONAL BENCHMARK

$\begin{array}{cc}\text { Mandatory } & \text { Optional } \\ 4 \mathrm{hr} & >4 \mathrm{hr}+\end{array}$

Basic Certification (BB, MT)

Certified Quality Auditor (CQA)

\section{PERFORMANCE AREA}

Mandatory

Supply Chain Process

Train-The-Trainer Certification

Review \& Validation of Procedures

Galileo (Automated Blood Analyzer)

Special Project (one)

Parathyroid

QC/PM (Daily, Weekly, Monthly)

Equipment Management \& Validation

Charge Technologist Responsibility

Review Orders

Reporting Problems

Problem Solving

Physician Interaction

Ensure Schedules are followed, make adjustments as necessary

Minimum of two (2) of the following:

NAT (counts as 2)

ORTHO Diagnostic (counts as 2)

BIOARRAY (counts as 2)

Flow Cytometry

Rapid Plasma Reagin testing (RPR), Cytomegalo Virus testing (CMV), SickleDex

Send Out testing procedures

Infectious Disease Specimen Processing

Bacti-Alert (Bacterial testing)

\section{OPTIONAL PERFORMANCE AREA}

2 Areas (Based on Business Needs)

RPR, CMV, SickleDex

Send Outs

Blood Distribution

Component Manufacturing

Donor Operations

SME (1 System)

Development of SOPs/Special Projects

Quality Assurance

Immunohematology Reference

Deviation Investigation 
Table 3. Competency Requirements in the Maturation Phase of the Professional Development Model

\section{EDUCATIONAL BENCHMARK \\ Mandatory $4 \mathrm{hr}$ \\ Basic Certification \\ (BB, MT, CQA)}

laboratory manager or designee will also be responsible for completing an annual competency check for each MLS to ensure his/her competency levels are maintained. Lastly, the laboratory manager must approve projects selected by MLS or assign projects designated for completion in their respective PDM competency levels.

Table 4. Competency Requirements in the Expert Phase of the

\author{
PERFORMANCE AREA \\ Mandatory \\ Troubleshooting Systems \\ Quality Assurance \\ Flow Charts \\ Statistical Process Control \\ Check sheet \\ Fellows/MDs Teaching/Training \\ Development of Standard Operating Procedures \\ Special Projects (two) \\ SME (2 systems) \\ Blood Distribution \\ Component Manufacturing \\ Immunohematology Reference \\ Deviation Investigation
}

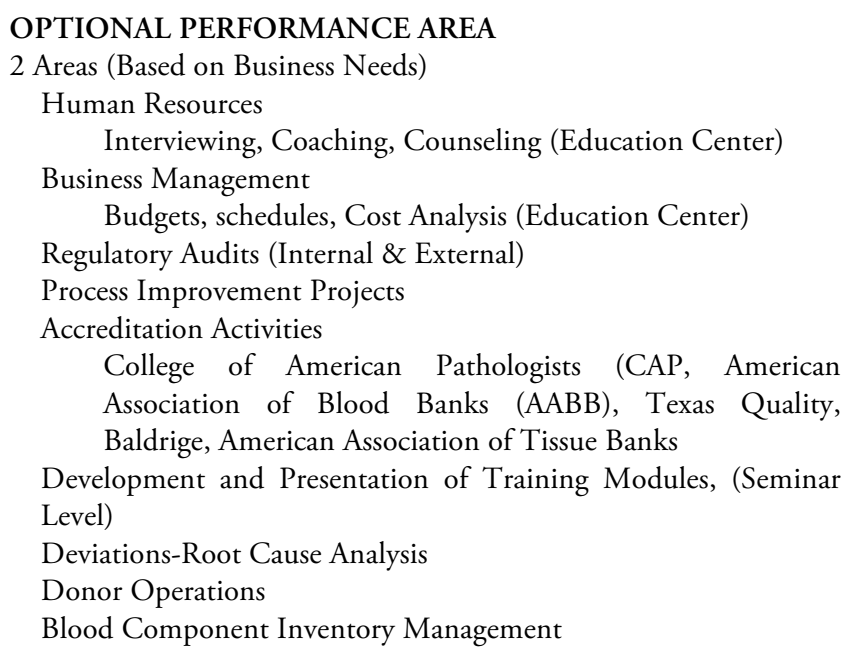

It is the sole responsibility of the MLS to complete each competency level through the Maturation level and to uphold the skills and knowledge throughout their entire employment period. The laboratory manager must approve the station that the employee proposes to begin training. Approval will be given on the basis of both the employee's preference and the needs of the laboratory. The laboratory manager or designee is also responsible for tracking the MLS progression through the PDM, ensuring that he/she has adequate time to train for and complete each competency level. Moreover, the Professional Development Model

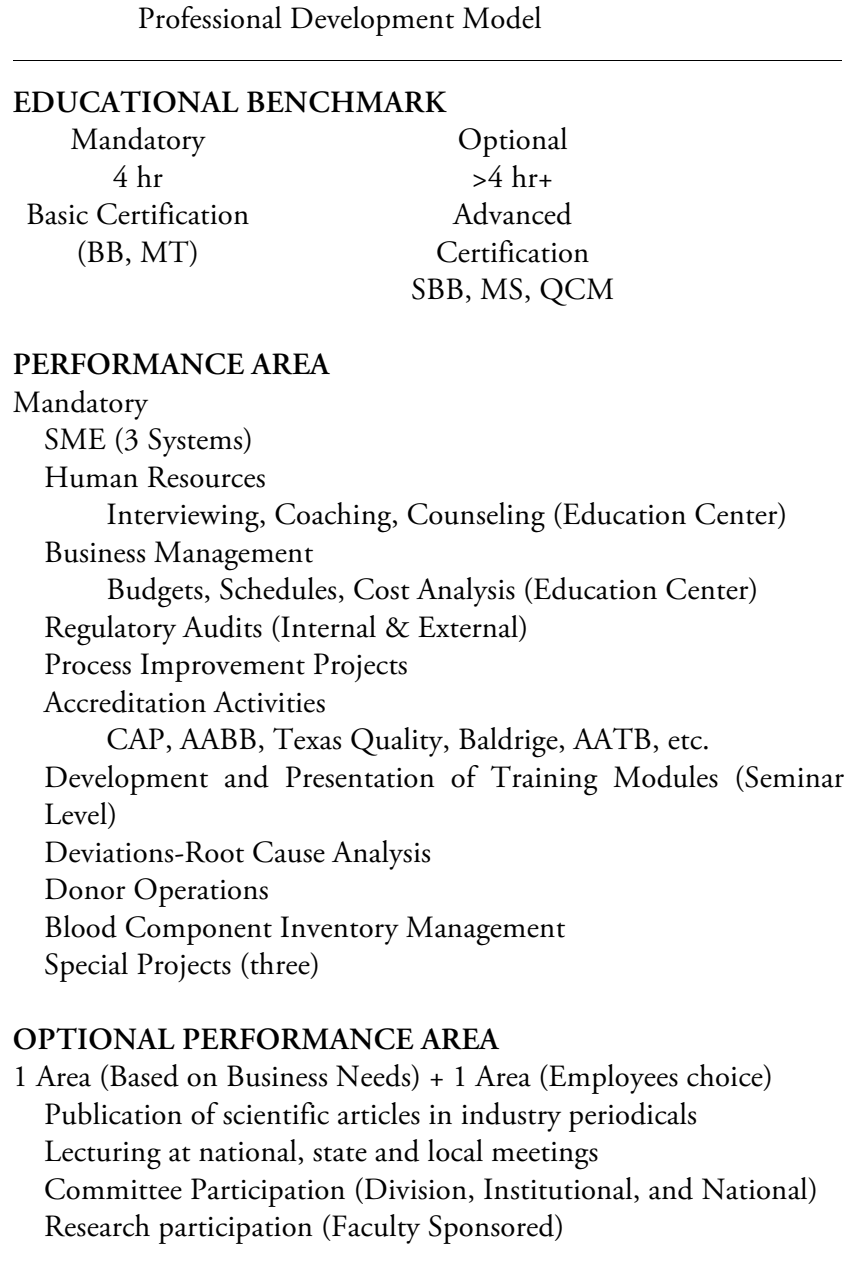

\section{Compensation}

Before compensation can be adjusted, the MLS must complete the assigned tasks for each competency level of the PDM in the time frame allowed. Any adjustments to a time frame allotted for completion of a competency level in the PDM must be made by department administrator or the laboratory manager. Compensation adjustment on the basis of completing PDM competency levels occurs twice a year at a time set by the laboratory manager. The options for compensation adjustment include a one-time bonus, an increase in base salary, or a mixture of the two on the basis of the 


\section{CLINICAL PRACTICE}

PDM level completed and the MLS current salary. All salary-related adjustments are made at the discretion of the laboratory administration and human resources department.

\section{DISCUSSION}

When implementing the PDM in the Immunohematology Laboratory at MD Anderson Cancer Clinic, we encountered several issues that should be mentioned as possible areas for improvement. The Immunohematology Laboratory has many employees, and at times it is difficult for one laboratory manager, or even several designees, to manage the PDMs for all the Medical Laboratory Scientists. We suggest that in the future, MLS who are further along in the PDM, mentor the MLS who are in the Discovery level to help reduce the laboratory managers burden. This suggested alteration will help the laboratory on many levels, but primarily enhance the teamwork and mentoring skills of all MLS, with the added benefit of more practically implementing the PDM. Another area for improvement may be the addition of two professional tracks. These tracks would be a technical track and a management track that would allow more options for the MLS to fit with his/ her professional motivations. These additions would also help create a more diverse and skilled laboratory.

\section{CONCLUSION}

The PDM described herein was developed to guide and reward the MLS as they progress through their professional journey in the medical laboratory science field of Immunohematology and help make the Section of Transfusion Medicine at MD Anderson Cancer
Clinic an exceptional learning environment. The PDM is an educational tool that covers laboratory processes and workflow management, which are two vital components of optimal operations in a clinical laboratory. In the promising future of laboratory medicine, a focus on professional training, expectations, and clearly outlined paths to promotion are essential to helping the MLS contribute to the field, while reaching their full potential and thereby improve the health of the patients it serves. ${ }^{3}$ Every person involved in patient care, from the laboratory to the bedside, should be held to a higher professional standard and should produce work of exceptional quality. We believe that this PDM not only fosters the growth of MLS at MD Anderson's Cancer Clinic Immunohematology Laboratory but can also serve as a useful tool for other clinical laboratories wanting to improve their laboratories professionalism and excellence.

\section{REFERENCES:}

1. Lennon PA, Lovshe D, Deleon E, Reynolds A, Pulido L, Cheong S, et al. Professional development model for cytogenetic technologists at The University of Texas MD Anderson Cancer Center sets high standards. J Assoc Genet Technol. 2010;36:195-7.

2. Lennon PA, Deleon E, Reynolds A, Pulido L, Lewing C, Mehta $\mathrm{P}$, et al. A model for rewarding professional growth in the diagnostic molecular oncology laboratory. J Assoc Genet Technol. 2011;37:80-3.

3. Bruns DE. Improving training in laboratory medicine. Clin Chim Acta. 2008;393:3-4

This article was supported in part by the National Institutes of Health through MD Anderson's Cancer Center Support Grant CA016672.

The peer-reviewed Clinical Practice Section seeks to publish case studies, reports, and articles that are immediately useful, are of a practical nature, or contain information that could lead to improvement in the quality of the clinical laboratory's contribution to patient care, including brief reviews of books, computer programs, audiovisual materials, or other materials of interest to readers. Direct all inquiries to Perry Scanlan, PhD, MT(ASCP), Medical Technology, Austin Peay State University, Room D212, Sundquist Science Complex, Box 4668, Clarksville TN 37044.

Clinical Laboratory Science encourages readers to respond with thoughts, questions, or comments regarding these articles. Email responses towestminsterpublishers@comcast.net. In the subject line, please type the journal issue and lead author such as "CLIN LAB SCI 25(1) RE HU". Selected responses may appear in the Dialogue and Discussion section in a future issue. Responses may be edited for length and clarity. We look forward to hearing from you. 